\title{
Antifungal Effectiveness of Various Intracanal Medicaments against Candida albicans: An In Vitro Study
}

\author{
Ganapa PV Srikumar ${ }^{1}$, R Shirish Kumar ${ }^{2}$, Siddharth Bardia ${ }^{3}$, Naiza Elsa Geojan ${ }^{4}$, Geeta Nishad ${ }^{5}$, Poonam Bhagat ${ }^{6}$
}

\begin{abstract}
Aim and objective: To evaluate the antifungal efficiency of various intracanal medicaments against Candida albicans.

Materials and methods: One-hundred and forty extracted human mandibular premolar teeth were decoronated, and the biomechanical preparation was done in crown-down technique. $10 \mu \mathrm{L}$ culture suspension of $C$. albicans was placed into the prepared root canal space of all the teeth. After 21 days of incubation, all the teeth were randomly divided into 7 groups with 20 teeth per each group. Group I: triple antibiotic powder (TAP) mixed with 3\% chitosan solution; group II: TAP mixed with macrogol-propylene (MP) glycol; group III: chlorhexidine-guttapercha (CHX-GP); group IV: Vitapex; group V: 2\% chlorhexidine gel; group VI: calcium hydroxide paste; group VII: normal saline with cotton (positive control) were used as intracanal medicaments, and the samples were incubated for 14 days. Intracanal medicaments were then completely removed using the canal brush. Dentinal chips were harvested from the walls of the root canal space in all samples using Gates-Glidden drills, were transferred into test tubes containing saline, and were serially diluted and placed in 140 Sabouraud dextrose agar plates, incubated at $37^{\circ} \mathrm{C}$ for 48 hours. Colony forming units (CFUs) of C. albicans were then counted using the digital colony counter.

Results: One-way ANOVA test showed statistically significant difference among the seven groups, as the $p$ value was $<0.001$. Tukey's post hoc test showed intergroup comparison between group I and group V; group II and group III were statistically nonsignificant as $p$ value was $>0.05$. Conclusion: $2 \%$ chlorhexidine gel and TAP mixed with $3 \%$ chitosan solution showed superior antifungal efficiency against C. albicans.

Clinical significance: Chitosan solution's inherent antifungal efficiency and slow and controlled drug release make it as an effective alternate carrier in mixing it with TAP instead of mixing TAP with MP.

Keywords: Candida albicans, Chitosan, Colony forming unit, Macrogol-propylene glycol, Triple antibiotic powder.

The Journal of Contemporary Dental Practice (2020): 10.5005/jp-journals-10024-2923
\end{abstract}

\section{INTRODUCTION}

Candida albicans are gram-positive yeast-like fungi, round or oval in shape, and are commonly found in $7-18 \%$ of failed root canal treatments due to persistent or secondary endodontic infections associated with recalcitrant periradicular lesions. So, the spectrum of antimicrobial activity of intracanal medicaments should include these microorganisms. ${ }^{1,2}$ The deeper penetration of C. albicans into the dentinal tubules of the root canals is due to its hyphal formation and thigmotropism property, and the phenotypic alteration of $C$. albicans helps in adapting to the ecologically harsh conditions of the root canal space. ${ }^{3}$ Elimination of microbes from the infected root canal system and prevention of reinfection is one of the fundamental aims of endodontic therapy, and the failure of endodontic treatment is due to the persistence of microbial flora in the root canal system. ${ }^{4}$ Disinfection of root canal space is an important step for the success of the root canal treatment. Intracanal medicaments are placed temporarily within the root canal space for few days to few weeks of time duration for root canal disinfection during endodontic treatment, and the medicaments with potent antifungal efficiency help in the successful management of persistent or secondary endodontic infections caused by C. albicans. ${ }^{3}$

Triple antibiotic powder (TAP) contains ciprofloxacin, metronidazole, and minocycline antibiotics, and the combined spectrum of antimicrobial activity and synergetic action of these three antibiotics overcomes microbial resistance and achieves higher antimicrobial efficiency. ${ }^{4,5}$ Hoshino et al. ${ }^{5}$ proposed mixing of MP glycol with TAP in a standard ratio of 1:7 and it can effectively eliminate all microorganisms present in the infected root canals, particularly those residing in the areas that cannot be reached even by the root canal irrigants. ${ }^{6}$

\begin{abstract}
1,5 Department of Conservative Dentistry and Endodontics, Triveni Institute of Dental Sciences, Hospital and Research Centre, Bilaspur, Chhattisgarh, India

${ }^{2}$ Department of Conservative Dentistry and Endodontics, Meghna Institute of Dental Sciences, Nizamabad, Telangana, India

${ }^{3}$ Department of Conservative Dentistry and Endodontics, Hithkarini Dental College and Hospital, Jabalpur, Madhya Pradesh, India

${ }^{4}$ Department of Conservative Dentistry and Endodontics, Pushpagiri College of Dental Sciences, Tiruvalla, Kerala, India

${ }^{6}$ Department of Pedodontics and Preventive Dentistry, Triveni Institute of Dental Sciences, Hospital and Research Centre, Bilaspur, Chhattisgarh, India
\end{abstract}

Corresponding Author: Ganapa PV Srikumar, Department of Conservative Dentistry and Endodontics, Triveni Institute of Dental Sciences, Hospital and Research Centre, Bilaspur, Chhattisgarh, India, Phone: +91 9685514220, e-mail: drsrikumar2611@gmail.com

How to cite this article: Srikumar GPV, Kumar S, Bardia S, et al. Antifungal Effectiveness of Various Intracanal Medicaments against Candida albicans: An In Vitro Study. J Contemp Dent Pract 2020;21(9):1042-1047.

Source of support: Nil

Conflict of interest: None

Chitosan is a natural copolymer obtained from chitin of crustaceans and shrimps shells; the deacetylation of chitin by alkaline substances yields in the formation of cationic aminopolysaccharide copolymer "chitosan." ${ }^{7}$ Chitosan solution is a versatile carrier or vehicle for drugs due to the presence of free

(c) The Author(s). 2020 Open Access This article is distributed under the terms of the Creative Commons Attribution 4.0 International License (https://creativecommons. org/licenses/by-nc/4.0/), which permits unrestricted use, distribution, and non-commercial reproduction in any medium, provided you give appropriate credit to the original author(s) and the source, provide a link to the Creative Commons license, and indicate if changes were made. The Creative Commons Public Domain Dedication waiver (http://creativecommons.org/publicdomain/zero/1.0/) applies to the data made available in this article, unless otherwise stated. 
amino groups, has an inherent antibacterial, antifungal action, helps in the local drug delivery with sustained effect of the drug, and was effectively used in the treatment of dentinal tubule infections. It is biocompatible and inexpensive, and the bioadhesion and biodegradable properties of chitosan are an added advantage for its intracanal use. ${ }^{8-10}$

Chlorhexidine-Guttapercha points are easy to place and remove from the root canal space and do not leave any residues. Chlorhexidine is released when CHX-GP comes in contact with moisture present in the root canal space. ${ }^{11} 2 \%$ chlorhexidine gel shows a phenomenon of substantivity, and it acts by permeating outer and inner plasma membrane of C. albicans causing precipitation of its cytoplasmic contents resulting in its cell death, thus effectively inhibiting the growth of C. albicans. ${ }^{3,12}$

The aim of this in vitro study was to evaluate the antifungal efficiency of TAP mixed with $3 \%$ chitosan solution, TAP mixed with MP glycol, 2\% chlorhexidine gel, CHX-GP, Vitapex, calcium hydroxide paste as intracanal medicaments on C. albicans.

\section{Materials and Methods}

The study was conducted in the Department of Conservative Dentistry and Endodontics, Triveni Institute of Dental Sciences and Hospital, Bilaspur, Chhattisgarh. One-hundred and forty freshly extracted human mandibular premolar teeth were collected. Inclusion criteria: noncarious, nonfractured, matured with closed root apices, nonrestored, single-rooted/single-root canal teeth. Exclusion criteria: carious, fractured, restored, multirooted, multicanaled, open root apex, defects within root portions, previously endodontically treated teeth.

The collected teeth were cleaned off from superficial debris, calculus, tissue tags, and stored in $10 \%$ formalin at $37^{\circ} \mathrm{C}$ and were used within 4 weeks of extraction. All teeth were decoronated at the cementoenamel junction with a diamond disc (DFS, Germany) attached to the slow-speed micromotor handpiece (NSK, Nakanishi Inc., Japan) to obtain uniform samples of approximately $12 \mathrm{~mm}$ in length. Necrotic pulp tissue was removed with barbed broach no. 15 (Dentsply, Malleifer, Tulsa Dental, Tulsa, USA). A no. $10 \mathrm{~K}$ (Kerr)file (Dentsply Maillefer, Ballaigues, Switzerland) was passively placed into each root canal until its tip was just visible at the apex, and the working length was established by subtracting 1 $\mathrm{mm}$ from this length. Biomechanical preparation was done using Nickel-Titanium rotary ProTaper Universal files (Dentsply, Maillefer, Switzerland) in the crown-down technique up to no. F2 file, and 17\% EDTA (ethylenediaminetetraacetic acid) (Rc Help Prime Dental Products, Pvt. Ltd., India), 3\% sodium hypochlorite solution (Safe Plus, Neelkanth Health Care, Pvt. Ltd., India), and distilled water were used for root canal irrigation. All teeth were then autoclaved at $121^{\circ} \mathrm{C}$ to ensure no microbial contamination.

Candida albicans [ATCC (American Type Culture Collection) 24433, Kwik Stik, Microbiologics, France] (Fig. 1) was used as the test strain. Under strict aseptic conditions, C. albicans was suspended in $5 \mathrm{~mL}$ of Sabouraud dextrose broth (HiMedia Laboratories Pvt. Ltd., India) and incubated at $37^{\circ} \mathrm{C}$ for 48 hours. The growth of $C$. albicans changes the turbidity of broth, and the optical density was adjusted to match the turbidity equivalent to 0.5 McFarland units $1.5 \times 10^{6} \mathrm{CFU} / \mathrm{mL}$. $10 \mu \mathrm{L}$ culture suspension of $C$. albicans was placed into the prepared root canal space of all samples using a sterile micropipette (Labtop, India) inside a laminar air flow cabinet (Bionics Scientific Technologies Pvt. Ltd., India) to prevent any airborne contamination, and the samples were then placed in

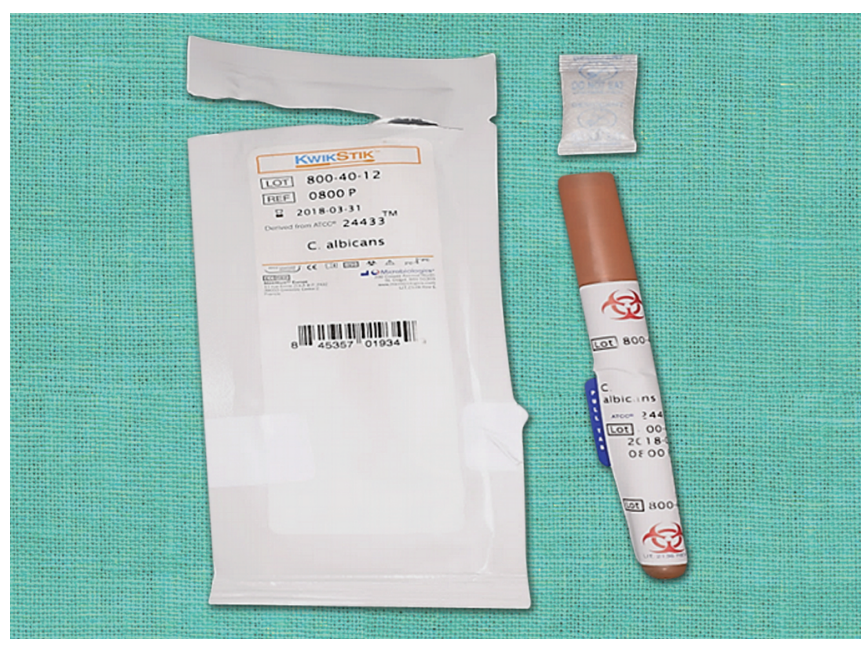

Fig. 1: Candida albicans (ATCC 24433, Kwik Stik, Microbiologics, France)

sterile test tubes, aerobically incubated (Zeal International, New Delhi, India) at $37^{\circ} \mathrm{C}$ for 21 days. All samples were then irrigated with $3 \mathrm{~mL}$ of distilled water to remove incubation broth, and the root canals were dried with sterile paper points (DiaDent Group International, Korea).

\section{Samples Classification}

All teeth were randomly divided into seven groups, with 20 teeth per each group depending on intracanal medicaments used. Group I:TAP mixed with $3 \%$ chitosan solution; group II:TAP mixed with MP glycol; group III: CHX-GP (Roeko Activ Points, Coltene Whaledent Pvt. Ltd., India); group IV: Vitapex (J Morita Corporation, Neo Dental Chemical Products Co. Ltd., India); group V: $2 \%$ chlorhexidine gel (Cavisept, Neelkanth health care, India); group Vl: calcium hydroxide paste (Rc Cal, Prime dental products, Pvt. Ltd., India); group VIII: normal saline with sterile cotton (positive control).

\section{Manipulation of TAP with 3\% Chitosan Solution}

Triple antibiotic powder was prepared by removing the film coating and crushing of antibiotic tablets: ciprofloxacin $500 \mathrm{mg}$ (Arion Healthcare Limited, Solan, India), metronidazole 400 mg (Jackson Laboratory Pvt. Ltd., Amritsar, India), and minocycline 100 mg (Pfizer Inc., Mumbai, India) separately using a hand mortar and pestle. The crushed antibiotic powders were then passed through a fine sieve to remove heavy filler particles, and a fine powder was obtained, which was then weighed separately and mixed in 1:1:1 proportion. ${ }^{5}$ $3 \%$ chitosan solution was prepared by dissolving $3 \mathrm{~g}$ of chitosan powder (HiMedia Laboratories Pvt. Ltd., India) in $100 \mathrm{~mL}$ of $0.1 \%$ acetic acid (HiMedia Laboratories Pvt. Ltd., India) in a glass beaker (Borosil, India) and mixed using a magnetic stirrer for over 2 hours to overcome the difficulty in dissolution of chitosan powder to obtain a homogenous clear solution. The obtained solution was then mixed with TAP in a dappen dish using stainless steel cement spatula in a standard ratio of $1: 7$ (3\% chitosan solution:TAP) into a creamy paste consistency.

Manipulation of TAP with MP glycol:TAP was mixed with MP glycol in standard ratio of 1:7 [MP glycol:TAP (1:1)] in a dappen dish using stainless steel cement spatula into a creamy paste consistency. ${ }^{5}$

Intracanal medicaments were placed in the root canals using Lentulo spiral instruments (Paste carriers, Mani, Inc., Japan) rotated in anticlockwise direction at $250 \mathrm{rpm}$ (revolutions per 
minute) attached to a slow-speed micromotor handpiece (NSK, Nakanishi Inc., Japan), and CHX-GP points were placed as per its manufacturer instructions. ${ }^{11}$ In the positive control group, sterile cotton moistened with normal saline (Amanta Health Care Ltd., Gujarat, India) was packed into the root canals using K(Kerr)-file no. 20 (Mani, Inc., Japan). All the samples were completely filled with respective intracanal medicaments as per their group, and the coronal access was restored with MD-Temp (META Biomed Co. Ltd., Korea) temporary restorative cement. The samples were then placed in sterile petri dishes, covered with damp sterile gauze, and incubated aerobically at $37^{\circ} \mathrm{C}$ for 14 days. Coronal temporary restorative cement was then removed from all samples. Using the medium-sized canal brush (Reoko Canal Brush, Coltene Whaledent $A G$, Switzerland) along with the use of $3 \mathrm{~mL}$ of distilled water per root canal, intracanal medicaments were completely removed from all samples, whereas CHX-GP points from group III and moistened cotton from positive control samples were removed using $\mathrm{H}$ (Hedstrom)-files no. 15,20 (Mani, Inc., Japan). The root canals were then dried with sterile paper points (Dia Dent, Dia Dent Group International, Korea).

Dentinal chips were collected from all the samples at a depth of $400 \mu \mathrm{m}$ using Gates Glidden drills Nos. 4 and 5 (Mani Inc., Japan), respectively, along the walls of the root canal space..$^{13}$ The collected dentinal chips from each sample were weighed using digital weighing balance, and only $2 \mathrm{mg}$ was transferred into each sterile test tube containing $1 \mathrm{~mL}$ of Sabouraud dextrose broth and incubated aerobically at $37^{\circ} \mathrm{C}$ for 48 hours; the contents of each test tube were then serially diluted, $100 \mu \mathrm{L}$ of broth in $100 \mu \mathrm{L}$ of sterile saline for 5 times up to $10^{-5}$ dilutions. $500 \mu \mathrm{L}$ of each dilution was then placed in one-hundred and forty Sabouraud dextrose agar plates (HiMedia Lab Pvt. Ltd., India) under strict aseptic conditions and incubated aerobically at $37^{\circ} \mathrm{C}$ for 48 hours. The agar plates were then checked for the growth of $C$. albicans and the number of CFUs was counted by digital colony counter (Secor, Scientific Engineering Corp., India) (Fig. 2) using the classical microbial counting technique, ${ }^{14,15}$ and the data were recorded.

\section{Results}

The obtained data were tabulated for statistical analysis using SPSS (Statistical Package for Social Sciences) computer software, version 19. The frequency of every score for each tested group

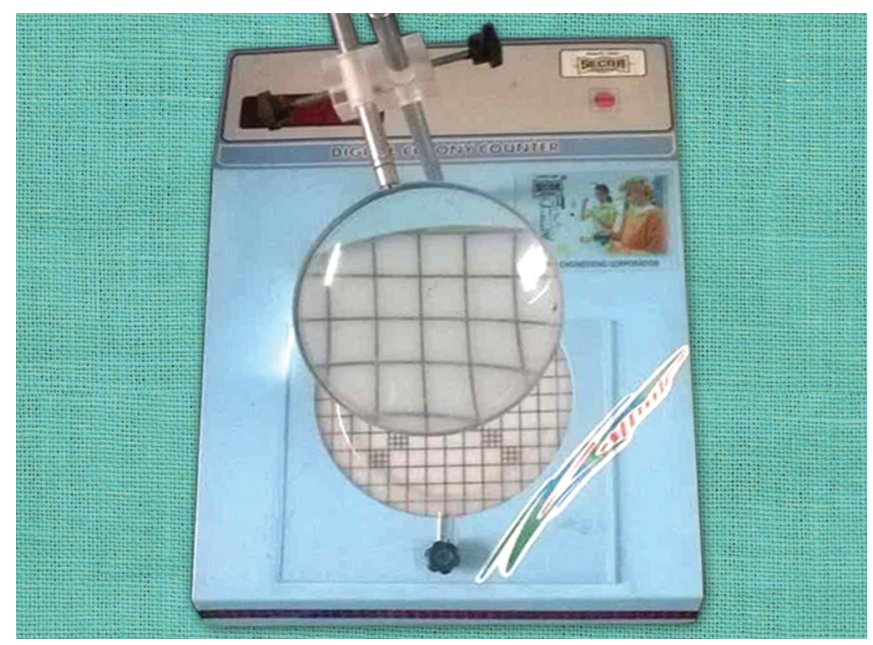

Fig. 2: Digital colony counter (SECOR, Scientific Engineering Corp, India) was counted to give descriptive analysis. The mean CFU values of group I were 21.90, group II: 45.40, group III: 42.90, group IV: 58.75, group V: 19.20, group VI: 77.95, group VII: 104.05, and the inferential statistical analysis was done using the one-way analysis of variance (ANOVA) test to determine if there was any statistically significant difference in the mean values and standard deviations of CFUs among seven groups, and it revealed statistically significant difference, as $p$ (probability) $<0.001$ (Table 1). Group I and group V showed least number of CFUs of $C$. albicans. group VI and group VII showed maximum number of CFU of C. albicans. Group II showed significant number of CFU of $C$. albicans compared to group I. To find any intergroup differences between the seven groups, Tukey's post hoc test was done and it showed intergroup comparison between group I and group V; group II and group III were statistically nonsignificant as the $p$ value was $>0.05$ (Table 2). Comparison of the mean CFU of $C$. albicans among the seven groups was shown in Figure 3. Two percent chlorhexidine gel and TAP mixed with $3 \%$ chitosan solution showed maximum antifungal efficiency and the calcium hydroxide paste showed poor antifungal efficiency as intracanal medicaments against C. albicans.

\section{Discussion}

The failure of endodontic treatment is due to the persistence of microorganisms in the complex anatomy of the root canal system even after effective biomechanical preparation and root canal irrigation. ${ }^{16}$ Constant attempts were made to find effective intracanal medicaments to eliminate $C$. albicans from the infected root canals. The use of a biocompatible intracanal medicament with effective antifungal properties will completely eliminate $C$. albicans from the root canal system and could significantly increase the success of the root canal treatment. ${ }^{17,18}$

The model proposed by Haapasalo and Orstavik ${ }^{19}$ was modified in the present study by adapting to the use of freshly extracted human teeth as samples rather than the previously used bovine teeth, and this modification was considered appropriate because of the marked difference in the diameter between the root canals of bovine and human teeth. ${ }^{20}$ Research studies on human extracted teeth would definitely be more suitable to simulate with clinical situations. ${ }^{21}$

In the present study, $3 \%$ chitosan solution was mixed with TAP in a standard ratio of 1:7. Chitosan powder is insoluble in water and also at neutral, alkaline $\mathrm{pH}$ values, but it is soluble in organic acids such as acetic acid. Acetic acid inhibits the growth of $C$. albicans at the concentration of $1 \%$ and higher, so the minimum concentration of acetic acid without any harmful or inhibiting effect on the viability of C. albicans was $0.1 \%{ }^{22}$ So, in the present study, $3 \mathrm{~g}$ of chitosan powder was mixed with $100 \mathrm{~mL}$ of $0.1 \%$ acetic acid to obtain $3 \%$ chitosan solution.

In the present study, the film coating over the antibiotics was completely removed, and only the active pharmacological ingredients were selected for the preparation, so that the film coating should not interfere with the formation of homogenous mixture of TAP.

In our study, TAP mixed with $3 \%$ chitosan solution showed superior antifungal efficiency against $C$. albicans, and this is in accordance with Jaheer Shaik et al. ${ }^{15}$ which showed superior antifungal efficiency of TAP mixed with chitosan solution on C. albicans. The cationically charged amino groups of chitosan combine with the anionic components such as $\mathrm{N}$-acetyl muramic acid, sialic acid, and neuramic acid on the cell surfaces of 
Table 1: One-way analysis of variance (ANOVA)

\begin{tabular}{|c|c|c|c|c|c|c|}
\hline Groups & Sample no. & $\begin{array}{l}\text { C. albicans CFU } \\
\text { (Mean } \pm S D)\end{array}$ & Fratio & $p$ value & $\begin{array}{l}\text { Significant groups at } \\
5 \% \text { level }\end{array}$ & $\begin{array}{l}\text { Nonsignificant } \\
\text { groups at } 5 \% \text { level }\end{array}$ \\
\hline Group I & $n=20$ & $21.90 \pm 2.38$ & 1916.80 & $<0.001(\mathrm{HS})$ & I vs II, III, IV, VI, VII & I vs V \\
\hline Group II & $n=20$ & $45.40 \pm 2.19$ & & & II vs I, IV, V, VI, VII & II vs III \\
\hline Group III & $n=20$ & $42.90 \pm 3.04$ & & & III vs I, IV, V, VI, VII & \\
\hline Group IV & $n=20$ & $58.75 \pm 2.01$ & & & IV vs I, II, III, V, VI, VII & \\
\hline Group V & $n=20$ & $19.20 \pm 1.40$ & & & V vs II, III, IV, VI, VII & \\
\hline Group VI & $n=20$ & $77.95 \pm 2.11$ & & & VI vs I, II, III, IV, V, VII & \\
\hline Group VII & $n=20$ & $104.05 \pm 6.10$ & & & VII vs I, II, III, IV, V, VI & \\
\hline
\end{tabular}

$p$, probability; SD, standard deviation; $F$, ratio of the between group variance to the within group variance; $\mathrm{HS}$, highly significant; $\mathrm{CFU}$, colony forming unit

Table 2: Tukey's post hoc test

\begin{tabular}{llllll}
\hline $\begin{array}{l}\text { Intergroup } \\
\text { comparison }\end{array}$ & Group II & Group III & Group IV & Group V & Group VI \\
\hline Group I & $p<0.01$ & $p<0.01$ & $p<0.01$ & $p>0.05$ & $p<0.01$ \\
Group II & & $p>0.05$ & $p<0.01$ & $p<0.01$ & $p<0.01$ \\
Group III & & $p<0.01$ & $p<0.01$ & $p<0.01$ & $p<0.01$ \\
Group IV & & & $p<0.01$ & $p<0.01$ & $p<0.01$ \\
Group V & & & & $p<0.01$ & $p<0.01$ \\
Group VI & & & & & $p<0.01$ \\
\hline
\end{tabular}

p, probability

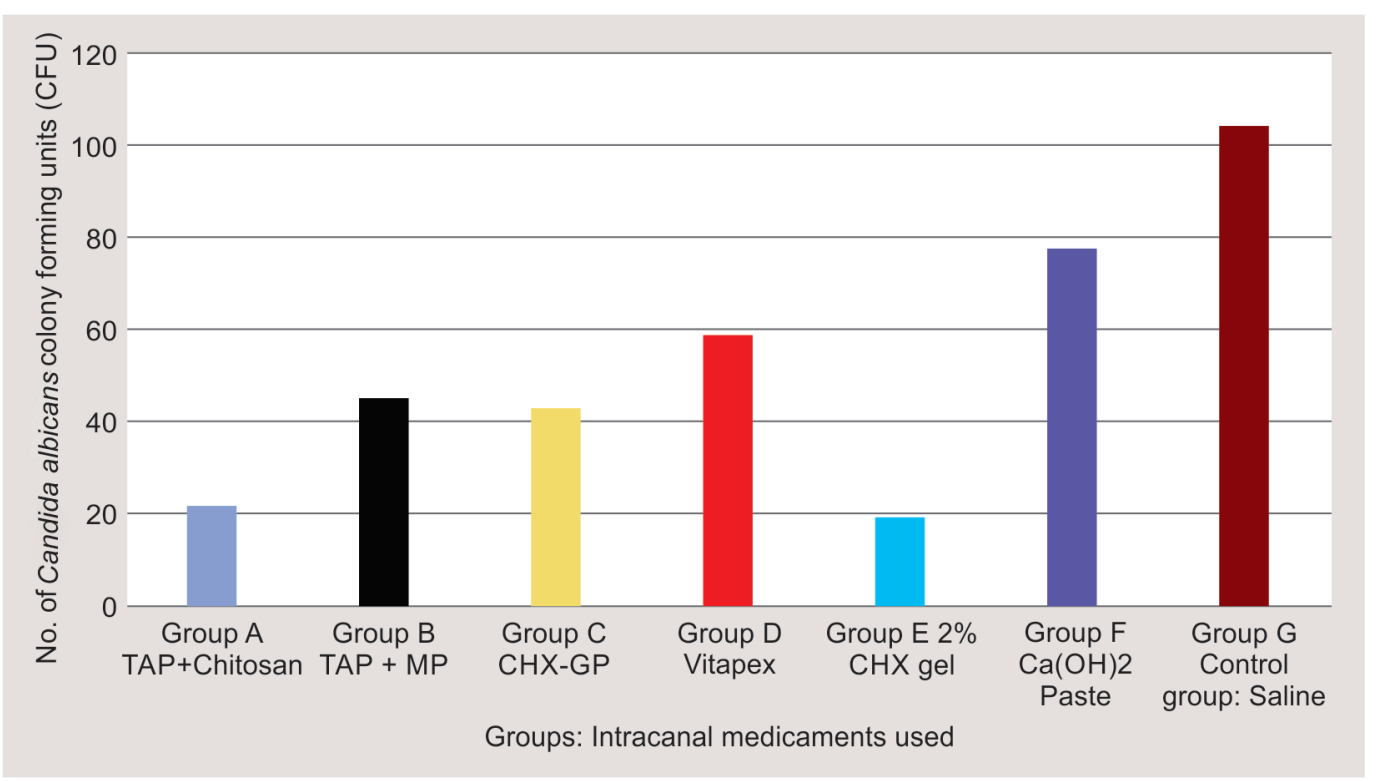

Fig. 3: Comparison of the mean CFU of C. albicans among the seven groups

microorganisms and suppress their growth by impairing the chelating transition of metal ions and inhibiting enzymes. ${ }^{14}$ So, in the present study, TAP was mixed with chitosan solution in an attempt to test the potential additive or synergistic effect on the viability of $C$. albicans.

William et al. ${ }^{23}$ concluded in their study that $90 \%$ of microorganisms remained positive following the root canal irrigation with $10 \mathrm{~mL}$ of $1.25 \%$ sodium hypochlorite solution. However, this dropped to $30 \%$ following the application of the TAP + MP for 2 weeks. Ordinola-Zapata et al. ${ }^{24}$ reported in their study that $\mathrm{TAP}+\mathrm{MP}$ was most effective at killing the microorganisms including C. albicans in the biofilms on the intraorally infected dentin model in comparison with $2 \%$ chlorhexidine gel and calcium hydroxide. Pramila et al. ${ }^{25}$ in their experimental study on dogs reported that intracanal drug delivery of TAP + MP resulted in $>99 \%$ reduction in mean CFU levels of $C$. albicans.

But, in contrary, in the present study TAP + MP showed minimal antifungal efficiency against $C$. albicans, and it was also found that the antifungal efficiency of TAP + MP was poor compared to TAP $+3 \%$ chitosan solution. TAP $+3 \%$ chitosan solution was found to be more effective inhibiting the growth of $C$. albicans compared to TAP + MP. Chitosan solution when used as a drug carrier or vehicle helps in the slow and controlled drug release improving its solubility, stability, and enhancing efficacy with reduced toxicity. ${ }^{26}$ 
So, the chitosan solution can effectively replace MP glycol as vehicle or carrier with TAP.

In our study, CHX-GP exhibited significant antifungal action on C. albicans, but it was found to be inferior compared to TAP mixed with $3 \%$ chitosan solution and $2 \%$ chlorhexidine gel, and our findings coincided with the studies of Ebert et al ${ }^{11}$ and Meltem et al., ${ }^{27}$ which confirmed poor antifungal action of CHX-GP on C. albicans compared to $2 \%$ chlorhexidine gel.

Vitapex as intracanal medicament in the present study showed poor antifungal efficiency on C. albicans, and it was partially explained by the fact that calcium hydroxide, an ingredient in Vitapex, interferes with the antiseptic capacity of dyadic combinations of intracanal medicaments. ${ }^{28}$ Contradicting to this, Fava et al. ${ }^{29}$ reported that Vitapex which contains silicone oil as its vehicle is less soluble and better diffuses into dentinal tubules resulting in superior antifungal efficacy against $C$. albicans.

$2 \%$ chlorhexidine gel exhibited maximum antifungal efficiency against $C$. albicans compared to all the other intracanal medicaments used in the present study, and similar results were presented by Mejia et al., ${ }^{30}$ Vaghela et al.,r ${ }^{21}$ Ercan et al.,r ${ }^{31}$ concluding superior antifungal efficiency of $2 \%$ chlorhexidine gel against C. albicans compared to calcium hydroxide paste, Vitapex, CHX-GP, and TAP mixed with MP.

In our study, calcium hydroxide paste showed the least antifungal efficiency on C. albicans compared to all the other intracanal medicaments used except the control group. Calcium hydroxide paste mostly depends on its alkalinity which is resisted by the proton pump property of $C$. albicans, and the dentin buffering potential can reduce the $\mathrm{pH}$ of calcium hydroxide paste thereby reducing its antifungal efficiency. ${ }^{32}$

To maximize the antimicrobial properties of intracanal medicaments, it is important to have dense homogenous filling of intracanal medicament in the root canal space. ${ }^{33}$ So, in the present study, Lentulo-spiral instruments were used to densely fill the root canal spaces with intracanal medicaments. Kozak et al. ${ }^{34}$ and Garip et al. ${ }^{35}$ reported that the canal brush was more effective in the removal of intracanal medicaments even from the narrowest areas of the root canal space due to its better contact. The canal brush is a highly flexible endodontic microbrush used manually in a rotary action, but it is more efficacious in its use when attached to a contra-angle handpiece. ${ }^{36}$ So, in the present study, the canal brush was used for the removal of intracanal medicaments.

In vitro studies definitely cannot duplicate the environment that exists in vivo. However, these in vitro evaluations provide information that aids clinicians in selecting the most suitable intracanal medicaments for specific clinical situations.

\section{Conclusion}

$2 \%$ chlorhexidine gel and TAP mixed with $3 \%$ chitosan solution showed maximum antifungal efficiency against $C$. albicans. Triple antibiotic powder mixed with $3 \%$ chitosan solution showed superior antifungal efficiency against $C$. albicans compared to TAP mixed with MP glycol, so $3 \%$ chitosan solution can be considered as a more effective alternate carrier or vehicle with TAP. The inherent antifungal and biodegradable properties and the slow and controlled drug release of chitosan are added advantages for its use as a vehicle even with other intracanal medicaments in the root canal treatment.

\section{References}

1. Chua EG, Parolia A, Ahlawat P, et al. Antifungal effectiveness of various intracanal medicaments against Candida albicans: an ex-vivo study. BMC Oral Health J 2014;14(53):1-8.

2. Sinha N, Patil S, Dodwad PK, et al. Evaluation of antimicrobial efficacy of calcium hydroxide paste, chlorhexidine gel and a combination of both as intracanal medicament. An in vivo comparative study. J Conserv Dent 2013;16(1):65-70.

3. Mohammadi Z, Abbott PV. The properties and applications of chlorhexidine in endodontics. Int Endod J 2009;42:288-302.

4. Brunton LL, Lazo JS, Parker KL, et al. The text book of pharmacological basis of therapeutics. 11th ed., New York: McGraw Hill Companies; 2006. pp. 1126-1182.

5. Hoshino E, Kurihara-Ando N, Sato I, et al. In vitro antibacterial susceptibility of bacteria from infected root dentin to a mixture of ciprofloxacin, metronidazole and minocycline. Int Endod J 1996;29:125-130.

6. Blackwell S, Cruz EV. Penetration of propylene glycol into dentine. Int Endod J 2002;35:330-336.

7. Pimenta JA, Zaparolli D, Pecora JD, et al. Chitosan: effect of a new chelating agent on the microhardness of root dentin. Braz Dent J 2012;23:212-217.

8. Illum L. Chitosan and its use as a pharmaceutical excipient. J Pharmaceut Res 1998;15(9):1326-1331.

9. Ballal NV, Kundabala M, Bhat KS, et al. Susceptibility of Candida albicans and Enterococcus faecalis to chitosan, chlorhexidine gluconate and their combination in-vitro. Aust Endod J 2009;35:2933.

10. Shrestha A, Kishen A. The effect of tissue inhibitors on the antibacterial activity of chitosan nanoparticles and photodynamic therapy. J Endod 2012;38:1275-1278.

11. Ebert J, Roggendorf MJ, Frank K. Antimicrobial activity of various 'active' gutta-percha points against Enterococcus faecalis in simulated root canals. Int Endod J 2008;41:249-257.

12. McDonnell G, Russell AD. Antiseptics and disinfectants: activity, action and resistance. Clin Microbiol Rev J 1999;12:147-149.

13. Krithikadatta J, Indira R, Dorothykalyani AL. Disinfection of dentinal tubules with $2 \%$ chlorhexidine, $2 \%$ metronidazole, bioactive glass when compared with calcium hydroxide as intracanal medicaments. J Endod 2007;33:1473-1476.

14. Collins $\mathrm{CH}$, Lyne PM, Grange JM, et al. Collins and Lyne's microbiological methods. ch.10. , Estimating microbial numbers. 8th ed., Oxford, London: Butterworth Heinemann; 2004. pp. 144-155.

15. Shaik J, Garlapati R, Nagesh B, et al. Comparative evaluation of antimicrobial efficacy of triple antibiotic paste and calcium hydroxide using chitosan as carrier against Candida albicans and Enterococcus faecalis: an in vitro study. J Conserv Dent 2014;17:335-339.

16. Agrawal V, Rao MSR, Dhingra K, et al. An in vitro comparision of antimicrobial efficacy of three root canal irrigants-Biopure MTAD, 2\% chlorhexidine gluconate and $5.25 \%$ sodium hypochlorite as a final rinse against E. faecalis. J Contemp Dent Pract 2013;14(5):842-847.

17. Radeva EN, Uzunov TT, Vacheva RS. In-vitro study of the antibacterial activity of calcium hydroxide on Candida albicans. Int J Sci Res 2014;3(9):2303-2306.

18. Kandaswamy D, Venkateshbabu N, Gogulnath D. Dentinal tubule disinfection with $2 \%$ chlorhexidine gel, propolis, morinda citrifolia juice, $2 \%$ povidone iodine, and calcium hydroxide. Int Endod J 2010;43:419-423.

19. Haapasalo M, Orstavik D. In vitro infection and disinfection of dentinal tubules. J Dent Res 1987;66:1375-1379.

20. Basrani B, Santos JM, Tjaderhane $L$, et al. Substantive antimicrobial activity in chlorhexidine treated human root dentin. Oral Surg Oral Med Oral Pathol Oral Radiol Endod 2002;94:240-245.

21. Vaghela DJ, Kandaswamy D, Venkateshbabu N, et al. Disinfection of dentinal tubules with two different formulations of calcium hydroxide as compared to $2 \%$ chlorhexidine: as intracanal medicaments against 
Enterococcus faecalis and Candida albicans: an in-vitro study. J Conserv Dent 2011;14(2):182-186.

22. Alburquenque C, Bucarey SA, Carrillo. AN. Antifungal activity of low molecular weight chitosan against clinical isolates of Candida species. J Med Mycol 2010;48(4):1018-1023.

23. William W, Teixeira F, Levin L, et al. Disinfection of immature teeth with a triple antibiotic paste. J Endod 2005;31:439-443.

24. Ordinola-Zapata R, Bramante CM, Minotti PG, et al. Antimicrobial activity of triantibiotic paste, $2 \%$ chlorhexidine gel and calcium hydroxide on an intraoral-infected dentin biofilm model. J Endod 2013;39:115-118.

25. Pramila R, Muthu M. Regeneration potential of pulp-dentin complex: systematic review. J Conserv Dent 2012;15:97-103.

26. Raafat D, Sahl HG. Chitosan and its antimicrobial potential - a critical literature survey. J Microb Biotechnol 2009;2:186-201.

27. Oztan MD, Kiyan M, Gerceker D. Antimicrobial effect, in vitro, of gutta-percha points containing root canal medications against yeasts and Enterococcus faecalis. Oral Surg Oral Med Oral Pathol Oral Radiol Endod 2006;102(3):410-416.

28. Seow WK. The effects of dyadic combinations of endodontic medicaments on microbial growth inhibition. J Pediatr Dent 1990;12(3):292-297.

29. Fava LR, Saunders WP. Calcium hydroxide pastes: Classification and clinical indications. Int Endod J 1999;32:257-282.
30. Carbajal Mejia JB. Antimicrobial effects of calcium hydroxide, chlorhexidine and propolis on Enterococcus faecalis and Candida albicans. J Investig Clin Dent 2013;4:1-7.

31. Ercan E, Dalli M, Dulgergil T, et al. Invitro assessment of the effectiveness of chlorhexidine gel and calcium hydroxide paste with chlorhexidine against Enterococcus faecalis and Candida albicans. Oral Surg Oral Med Oral Pathol Oral Radiol Endod 2006;102(2): 27-31.

32. Menezes MM, Valera MC, Jorge AOC. In vitro evaluation of the effectiveness of irrigants and intracanal medicaments on microorganisms within root canals. Int Endod J 2004;37:311-319.

33. Ranga Reddy DS, Shankar Narayan G, Venkatesan SM. Qualitative and quantitative analysis of intracanal delivery systems of calcium hydroxide: an in vitro study. J Sci Dent 2013;3(1):8-14.

34. Kozak A, Roggendort MJ, Ebert J. Efficiency of cleaning procedures to remove chlorhexidine calcium hydroxide paste in root canal extensions. Int Endod J 2009;42(1):33-36.

35. Garip Y, Sazak H, Gunday M. Evalution of smear layer removal after use of canal brush: an SEM study. Oral Surg Oral Med Oral Pathol Oral Radiol Endod 2010;110(2):62-66.

36. Gorduysus M, Yilmaz Z, Gorduysus O, et al. Effectiveness of a new canal brushing technique in removing calcium hydroxide from the root canal system: a scanning electron microscope study. J Conserv Dent 2012;15(4):367-370. 